\title{
Thickening of Glomerular Basement Membrane in Spontaneously Diabetic Rats
}

\author{
S. Yagihashi, Y. Goto, M. Kakizaki, and N. Kaseda \\ First Department of Pathology and Third Department of Internal Medicine, Hirosaki University School of Medicine, Hirosaki \\ and Third Department of Internal Medicine, Tohoku University School of Medicine, Sendai, Japan
}

\begin{abstract}
Summary. The glomerular basement membrane of spontaneously diabetic rats was investigated by quantitative analysis using electron microscopy, with special reference to the effect of ageing. Constant agerelated increase in the width of basement membrane was ascertained both in diabetic and control rats, and the mean values of basement membrane thickness were always higher in the spontaneously diabetic rats than in normal control rats. Significant thickening of glomerular basement membrane was found in the diabetic rats at 12 weeks of age, while younger diabetic rats had no definite increase. The difference in basement membrane thickness between diabetic and normal control rats became larger with increasing age.
\end{abstract}

Key words: Spontaneous diabetes rats, glomerular basement membrane, ageing, quantitative analysis.

Rats with spontaneous diabetes can be obtained by selective breeding of animals with slightly abnormal glucose tolerance, selected from a population of normal Wistar rats $[1,2]$. These rats show low insulin secretion, glycosuria and glucose intolerance. We have demonstrated previously a significant increase in glomerular basement membrane thickness in these diabetic rats at 24 weeks of age when compared with age-matched control rats [3]. However, no definitive study has been made of the age or stage of the disease when the increase of the capillary basement membrane thickness becomes apparent. The present study was undertaken to clarify these points.

\section{Materials and Methods}

Twenty two spontaneously diabetic rats and 36 normal rats of male Wistar strain were used. Diabetic rats were divided into six groups; three 8 week-old, three 12 week-old, two 16 week-old, six 24 week-old, four 25 to 28 week-old and four 29 to 32 week-old. Nine groups of age-matched control rats consisted of animals from four to 36 weeks of age at four weeks intervals. Each control group consisted of four rats. All rats were given glucose tolerance tests $(2 \mathrm{~g} / \mathrm{kg}$, orally). Blood glucose determination was made using a glucose oxidase method, as described elsewhere [1,2]. The rats were given water and standard rat chow (Oriental MF) libitum until sacrifice. Detailed laboratory data and glomerular basement membrane thickness of normal control rats have been published in our previous report [4].

Tissue specimens for electron microscopy were prepared as follows: after an overnight fast, rats were killed by decapitation. The abdominal cavity was opened, and the left renal cortex cut into small pieces. The small specimens of renal tissue were immersed in $4 \mathrm{~g} / 100 \mathrm{ml}$ glutaraldehyde in $0.15 \mathrm{~mol} / 1$ phosphate buffer, $\mathrm{pH} 7.4$, for $1 \frac{1 / 2}{\mathrm{hrs}}$ and rinsed in the same buffer overnight. After postfixation in $1 \mathrm{~g} / 100 \mathrm{ml}$ osmium tetroxide, specimens were dehydrated through an ascending series of ethanol. They were then embedded in Maraglas 655 and polymerized at $60^{\circ} \mathrm{C}$ for 48 hrs [5]. Ultrathin sections were obtained by Porter-Blum MT 2B ultramicrotome and stained with uranyl acetate and lead citrate [6]. Electron micrographs were obtained using a Hitachi HU $12 \mathrm{~S}$ electron microscope.

For the quantitative analysis, electron micrographs were taken at a magnification of $6,000 \times$ and developed to a final magnification of $15,000 \times$. The micrographs for the measurements of glomerular basement membrane thickness were selected at random, and the person who estimated the basement membrane thickness was not aware of the objectives of the study. The method for the measurements of glomerular basement membrane thickness was based on that of Osawa et al. [7]. Sites for measurements were selected only from peripheral parts of the glomerular capillary loops which were clearly separate from mesangial regions. The peripheral glomerular basement membrane was measured at one micron intervals. Only vertical sections clearly cut through both epithelial and endothelial cell membranes were selected for the measurements. The width of basement membrane was measured 
Table 1. Body weight (BW), blood glucose during glucose tolerance test and glomerular basement membrane thickness (GBMT) in experimental rats

\begin{tabular}{|c|c|c|c|c|c|c|c|c|c|}
\hline \multirow[t]{2}{*}{ Group } & \multirow{2}{*}{$\begin{array}{l}\text { Age } \\
\text { (wks) }\end{array}$} & \multirow[t]{2}{*}{ (No) } & \multirow{2}{*}{$\begin{array}{l}\text { BW } \\
(\mathrm{g})\end{array}$} & \multicolumn{5}{|c|}{ Blood glucose $(\mathrm{mg} / 100 \mathrm{ml})$} & \multirow{2}{*}{$\begin{array}{l}\text { Mean GBMT } \\
(\AA)\end{array}$} \\
\hline & & & & Fast & $30^{\prime}$ & $60^{\prime}$ & $90^{\prime}$ & $120^{\prime}$ & \\
\hline \multirow[t]{9}{*}{ Control $^{a}$} & 4 & (4) & $109 \pm 14$ & $84 \pm 5$ & $173 \pm 18$ & $140 \pm 19$ & $117 \pm 7$ & $101 \pm 2$ & $1190 \pm 68$ \\
\hline & 8 & (4) & $224 \pm 17$ & $91 \pm 3$ & $129 \pm 17$ & $134 \pm 9$ & $115 \pm 6$ & $107 \pm 7$ & $1291 \pm 46$ \\
\hline & 12 & (4) & $278 \pm 13$ & $80 \pm 5$ & $142 \pm 5$ & $133 \pm 12$ & $109 \pm 9$ & $98 \pm 5$ & $1354 \pm 35$ \\
\hline & 16 & (4) & $313 \pm 10$ & $86 \pm 5$ & $127 \pm 12$ & $123 \pm 6$ & $110 \pm 18$ & $99 \pm 17$ & $1473 \pm 13$ \\
\hline & 20 & (4) & $345 \pm 18$ & $87 \pm 6$ & $131 \pm 9$ & $121 \pm 4$ & $118 \pm 9$ & $103 \pm 6$ & $1541 \pm 35$ \\
\hline & 24 & (4) & $404 \pm 48$ & $90 \pm 8$ & $124 \pm 16$ & $127 \pm 8$ & $124 \pm 11$ & $117 \pm 11$ & $1605 \pm 38$ \\
\hline & 28 & (4) & $488 \pm 41$ & $85 \pm 6$ & $125 \pm 11$ & $120 \pm 9$ & $117 \pm 10$ & $108 \pm 9$ & $1846 \pm 65$ \\
\hline & 32 & (4) & $563 \pm 48$ & $84 \pm 8$ & $136 \pm 11$ & $122 \pm 15$ & $114 \pm 10$ & $101 \pm 7$ & $2053 \pm 72$ \\
\hline & 36 & (4) & $625 \pm 40$ & $89 \pm 1$ & $125 \pm 14$ & $122 \pm 1$ & $115 \pm 12$ & $109 \pm 4$ & $2383 \pm 114$ \\
\hline \multirow[t]{6}{*}{ Diabetic } & 8 & (3) & $200 \pm 10$ & $98 \pm 12$ & $228 \pm 41$ & $212 \pm 37$ & $162 \pm 22$ & $128 \pm 14$ & $1386 \pm 72$ \\
\hline & 12 & (3) & $263 \pm 15$ & $105 \pm 8$ & $233 \pm 29$ & $231 \pm 20$ & $186 \pm 21$ & $158 \pm 11$ & $1540 \pm 100^{\mathrm{b}}$ \\
\hline & 16 & (2) & $298 \pm 40$ & $96 \pm 11$ & $213 \pm 41$ & $192 \pm 28$ & $177 \pm 24$ & $155 \pm 15$ & $1735 \pm 155^{\mathrm{b}}$ \\
\hline & 24 & (6) & $387 \pm 56$ & $95 \pm 19$ & $220 \pm 42$ & $223 \pm 70$ & $212 \pm 70$ & $176 \pm 59$ & $2004 \pm 121^{b}$ \\
\hline & $25-28$ & (4) & $396 \pm 98$ & $92 \pm 6$ & $195 \pm 30$ & $214 \pm 22$ & $226 \pm 41$ & $199 \pm 40$ & $2273 \pm 107^{b}$ \\
\hline & $29-32$ & (4) & $453 \pm 75$ & $108 \pm 10$ & $185 \pm 59$ & $183 \pm 47$ & $173 \pm 34$ & $155 \pm 29$ & $2488 \pm 242^{b}$ \\
\hline
\end{tabular}

$\mathrm{M} \pm \mathrm{SD}$ a Detailed control data has been published in elswhere [4]

b $\mathrm{p}<0.01$ (vs. control)

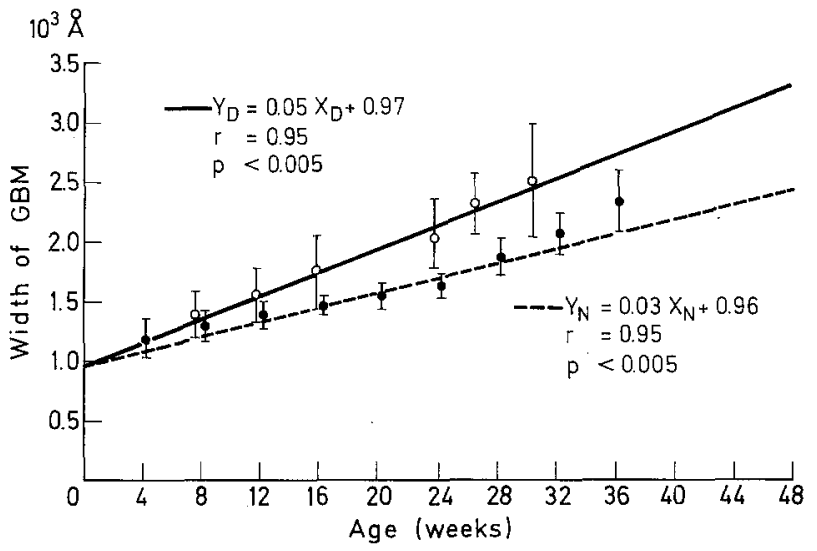

Fig. 1. The relationship between age and width of glomerular basement membrane (GBM) in the spontaneously diabetic rats (regression equation shown by solid line) and normal control rat (broken line). Significant thickening of basement membrane appeared after the age of 12 weeks. $\frac{\hat{T}}{\mathrm{~S}}$ stands for mean thickness of $\mathrm{GBM} \pm 2 \mathrm{SD}$ of spontaneously diabetic rats, while for that of control rats

with a micrometer. The thickness of basement membrane obtained by direct measurements on the photographs was converted into Angstrom units according to the calculated magnifications. Two hundred and sixty sites from two or three glomeruli in each rat, totalling 15,080 sites $(5,720$ sites in diabetic rats and 9,360 sites in normal control rats) in 58 rats were measured.

The comparisons between rats, or groups of rats, and the statistical evaluation by Student's t-test were based on mean glomerular basement membrane thickness for each individual rat. Statistically significant differences in values between diabetic and control groups were sought in the following ways. First, each rat was compared within their own groups of diabetic, or age-matched control rats respectively. In this case almost insignificant differences were obtained. However, a significant difference could be demonstrated on group comparisons, that is, between the diabetic rats and agematched control rats.

The measurements of basement membrane thickness in each individual animal were plotted as frequency distribution curves and are represented by mean values for each group.

\section{Results}

\section{Glucose Tolerance Test}

Spontaneously diabetic rats showed glucose intolerance (Table 1). Although almost all diabetic rats had a slight increase of fasting blood sugar concentration, the most characteristic features of the glucose tolerance tests were the high two hour glucose levels. The sum of blood glucose values at 5 points during the glucose tolerance test was over $650 \mathrm{mg} / 100 \mathrm{ml}$ in the diabetic rats, and some diabetic rats had values over $1,000 \mathrm{mg} / 100 \mathrm{ml}$, while normal control rats had sums from 500 to $650 \mathrm{mg} / 100 \mathrm{ml}$.

The body weight of the diabetic rats increased with ageing, although there were some lean rats in the older groups. However, no relationship between body weight and glucose intolerance was recognised.

\section{Quantitative Analysis of Glomerular Basement Membrane Thickness of Diabetic Rats}

Mean glomerular basement membrane thickness of the diabetic rats and age-matched control rats are summarised in the Table. Both in diabetic rats and control rats, a gradual and progressive age-related increase in basement membrane thickness was found. 

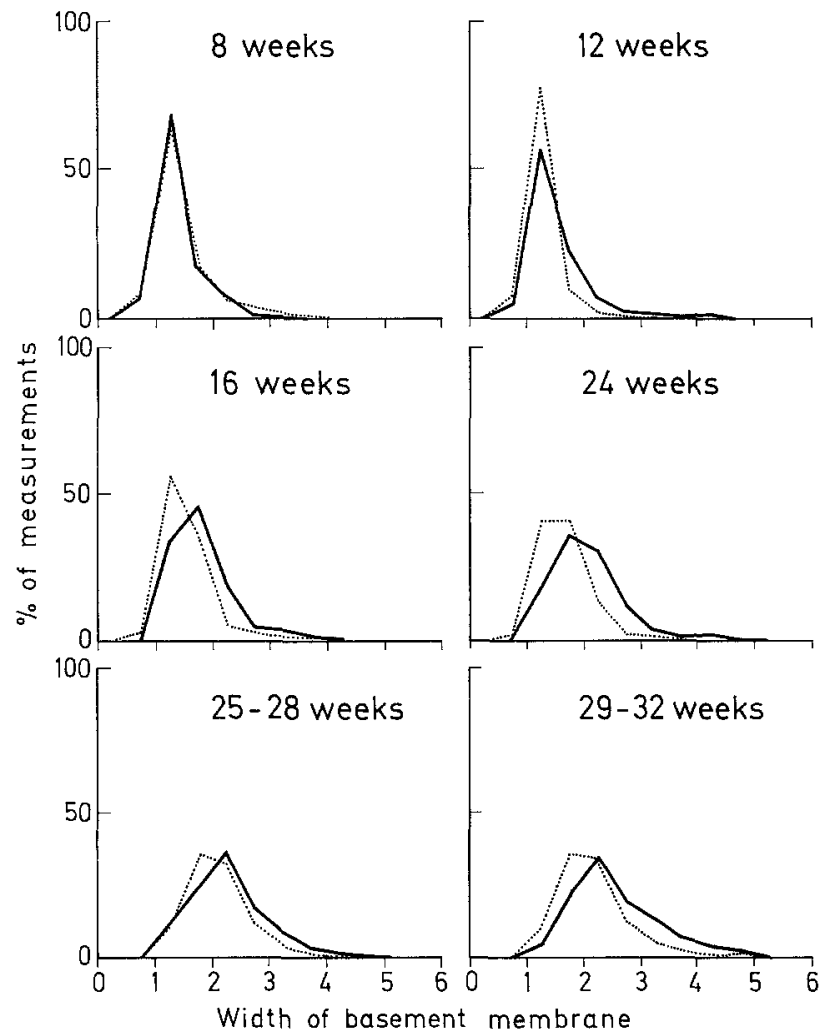

Fig. 2. Frequency distribution curves of measurements for glomerular basement membrane thickness of spontaneously diabetic rats (illustrated by solid lines). The curves are expressed by mean values of measurements. Broken lines show the curves of normal control rats

Definite thickening of basement membrane was detected in the diabetic rats by 12 weeks. Although there was no significant increase in the 8 week-old diabetic rats, the thickness of basement membrane in the diabetic rats was always higher than that of control rats at all time period. The differences between diabetic and control rats became more prominent with increasing age. The progression of basement membrane thickening with age in the diabetic rats was represented by the regression equation: $\mathrm{Y}=$ $0.05 \mathrm{X}+0.97, \mathrm{r}=0.95(\mathrm{p}<0.005)$, while that in the normal control rats was represented by $\mathrm{Y}=0.03 \mathrm{X}$ $+0.96, r=0.95(p<0.005)$, where $Y$ stands for the mean thickness of glomerular basement membrane [in $\AA \times 10^{3}$ ] and $\mathrm{X}$ is the age in weeks, as shown in Fig. 1. The standard deviation (SD) of basement membrane thickness was largest in the diabetic rats at 29 to 32 weeks of age. Frequency distribution curves of the basement membrane thickness from the diabetic rats showed a sharp peak at 8 and 12 weeks, that were almost identical to those of control rats, but became broader with increasing age (Fig. 2).

\section{Discussion}

Our rats with spontaneous diabetes have characteristic diabetic features; glycosuria, glucose intolerance, reduced insulin secretion and other biochemical properties [3]. In both normal and diabetic animals, constant age-related increase in glomerular basement membrane thickness has been reported by only a few workers $[8,9,10]$. The evolution of glomerular basement membrane thickening with increasing age and the age when the thickening becomes significantly greater in diabetic animals is still not known.

In this study, a progressive age-related increase in glomerular basement membrane width of spontaneously diabetic rats as well as age-matched control rats was well documented. The definite increased thickening of basement membrane appeared in the diabetic rats at 12 weeks of age. Thereafter, the extent of thickening increased with age.

The appearance of significant thickening of basement membrane occurred at an early age in the spontaneously diabetic rats when compared with other kinds of diabetic animals. In experimentally diabetic dogs, significant thickening of glomerular basement membrane was recognised at 2 to 3 years after alloxanisation [11]. While in spontaneous diabetic spiny mice, Orci et al. [9] found it at 24 weeks. They stated that the measurements of the thickness of glomerular basement membrane showed age-dependent increases perhaps accelerated in diabetic animals. On the other hand, genetically determined diabetic KK mice showed early manifestation of diffuse glomerulosclerosis at the age of two months Opperman et al. [12]. It was suggested by them that diabetic glomerulopathy may be regulated by diabetic genes. In human diabetics, Østerby Hansen [13] reported that recent onset juvenile diabetics showed no increase in the glomerular basement membrane thickness as compared with age-matched controls, whereas the diabetics with a longer history of diabetes showed a significant increase in glomerular basement membrane thickness [14]. Frequency distribution curves on the measurements of glomerular basement membrane thickness in human diabetics with a longer history of diabetes as they illustrated in their report, are very similar to those of our spontaneously diabetic rats.

Recent studies suggest that classical juvenile onset type diabetes in young people may be provoked by viral infection and be less related to the genetic background [15]. If this is the case, it will not be idiopathic(genetic) diabetes, but secondary (nongenetic) diabetes. Hence the question will arise whether the thickening of the capillary basement membrane occurs as a result of the diabetic state, or 
as one of the primary changes associated (perhaps genetically) with diabetes mellitus. The answer to this question may be obtained by studying spontaneously diabetic animals rather than by the studying druginduced or pancreatectomised experimental diabetic animals.

The results of the present study showed that the mean values of the thickness of glomerular basement membrane were always higher in the spontaneously diabetic rats than in the normal control rats throughout the period of observation (Fig. 1). The differences were significant after the 12 weeks. It is possible that differences before 12 weeks would have become significant if the number of the rats were large enough. In this case it would suggest that the thickening of the capillary basement membrane was a primary change probably unrelated to the hyperglycaemic state. The study of infant rats in very early stages of diabetes when the biochemical changes are minimal, should give an answer to this problem.

Acknowledgements. The authors are greatly thankful to Prof. Kazunori Nagai, First Department of Pathology, Hirosaki University School of Medicine for his thoughtful advices and criticism in preparation of this manuscript, and to Dr. Morimi Nishihira, Dr. Norihiko Shimoyama, Mr. Shun-ichi Takeuchi and Mr. Tomio Koizumi for their skillful technical assistances.

\section{References}

1. Goto, Y., Kakizaki, M., Masaki, N.: Spontaneous diabetes produced by selective breeding of normal Wistar rats. Proc. Jpn. Acad. 51, 80-85 (1975)

2. Goto, Y., Kakizaki, M., Masaki, N.: Production of spontaneous diabetic rats by repetition of selective breeding. Tohoku J. Exp. Med. 119, 85-90 (1976)

3. Goto, Y., Kakizaki, M., Toyota, T., Masaki, N., Kitahara, A., Yagihashi, S., Kimura, K.: Spontaneous diabetes produced by repeated selective breeding of normal Wistar rats. In: J.S. Bajaj (Ed.): Diabetes, pp. 703-710. Amsterdam: Excerpta Medica 1977

4. Yagihashi, S.: Quantitative estimation of age-related thickening of glomerular basement membrane in normal rats. Tohoku J. Exp. Med. 126, (1978) (in press)
5. Spurlock, B. O., Kattine, V.C., Freeman, J.A.: Technical modifications in Maraglas embedding. J. Cell Biol. 17, 203-207 (1963)

6. Reynolds, E. S.: The use of lead citrate at high $\mathrm{pH}$ as an electron opaque stain in electron microscopy. J. Cell Biol. 17, 208-212 (1963)

7. Osawa, G., Kimmelstiel, P., Seiling, V.: Thickness of glomerular basement membranes. Am. J. Clin. Pathol. 45, 7-20 (1966)

8. Shirai, T., Welsch, G. W. 3rd., Sims, E. A.: Diabetes mellitus in the Chinese hamster. II. The evolution of renal glomerulopathy. Diabetologia 3, 266-286 (1967)

9. Orci, L., Stauffacher, W., Amherdt, M., Pictet, R., Renold, A. E., Rouiller, Ch.: The kidney of spiny mice (Acomys cahirinus): Electron microscopy of glomerular changes associated with ageing and tubular glycogen accumulation during hyperglycaemia. Diabetologia 6, 343-355 (1970)

10. Like, A. A., Lavine, R. L., Poffenbarger, P. L., Chick, W. L.: Studies in the diabetic mutant mouse. VI. Evolution of glomerular lesions and associated proteinuria. Am. J. Pathol. 66, 193-224 (1972)

11. Bloodworth, J. M. B. Jr., Engerman, R. L., Camerini-Dávalos, R. A.: Variations in capillary basement membrane width produced by aging and diabetes mellitus. In: R. A. CameriniDávalos, H. Cole (Eds.): Advances in Metabolic Disorders, Suppl. 1: Early Diabetes, pp. 279-295. New York: Academic Press 1970

12. Opperman, W., Ehrenreich, T., Bloodworth, J.M. B., Jr., Camerini-Dávalos, R. A.: Small-vessel disease in diabetes mellitus. In: L. J. Kryston, R. A. Shaw (Eds.): Endocrinology and Diabetes Mellitus, pp. 427--442. New York: Grune \& Stratten Inc. 1975

13. Østerby Hansen, R.: A quantitative estimate of the peripheral glomerular basement membrane in recent juvenile diabetes. Diabetologia 1, 97-100 (1965)

14. Østerby Hansen, R., Lundbaek, K.: The basement membrane morphology in diabetes mellitus. In: M. Ellenberg, J. Rifkin (Eds.): Diabetes mellitus: Theory \& Practice, pp. 178-209. New York: McGraw-Hill Co. 1970.

15. Craighead, J. E.: Viral diabetes. In: E. W. Volk, K. F. Wellman (Eds.): The Diabetic Pancreas, pp. 467-488. New York: Plenum Press 1977

Received: January 3, 1978 ,

and in revised form: April 14, 1978

Dr. S. Yagihashi

The First Department of Pathology

Hirosaki University School of Medicine

Hirosaki 036

Zaifucho 5, Japan 